\title{
Exploring the Effect of Monetary Incentives on User Behavior in Online Sharing Platforms
}

\author{
Yixin $\mathrm{Lu}$ \\ George Washington University \\ yixinlu@gwu.edu
}

\author{
Carol X. J. Ou \\ Tilburg University \\ carol.ou@uvt.nl
}

\author{
Spyros Angelopoulos \\ Tilburg University \\ s.angelopoulos@uvt.nl
}

\begin{abstract}
We examine the impact of monetary incentives on user onboarding in online sharing platforms. Specifically, drawing upon the literature of monetary incentives, privacy, and consumer behavior, we conduct a randomized field experiment to explore users' initial engagement and interaction with an online car-sharing platform. Our empirical analyses show that monetary incentives are no better than simple email reminders in encouraging users' selfdisclosure of private information nor their active engagement with the platform (i.e., actual booking via the platform). Our work sheds new light on the heated debate over the design and deployment of monetary incentives in digital platforms, and provides useful implications for both academia and the industry.
\end{abstract}

\section{Introduction}

The internet-based markets play a critical role in today's economy. They have greatly increased the variety of product and service offerings, and improved the efficiency of the exchange of information, goods, services and payments [1]. Most recently, the proliferation of digital platforms, such as Airbnb and Uber, have made the sharing of physical assets much cheaper and easier than ever, giving rise to the socalled "sharing economy".

The companies under the umbrella of "sharing economy" are attracting increasing attention from both academics and practitioners, given their economic and social significance. Specifically, the "collaborative consumption" concept underlying the sharing economy, holds great potential in maximizing social welfare and contributing to environmental sustainability. Take cars for example. The possibility to rent a car in one's neighborhood could lead to forgo the ownership, and turn to on-demand services. This could potentially reduce the total number of cars on the streets and thereby alleviate the congestion $[2,3]$.
Despite these promises, the majority of the peer-topeer sharing platforms still face the fundamental challenge of how to attract more users on board and get them engaged in the sharing activities facilitated by the platform. While user onboarding processes are designed and implemented in different ways -some platforms have used a simple registration form followed by a greeting message sent by email, while others have chosen to use the social login option via popular social networking platforms such as Facebook, LinkedIn, and Twitter - given the nature of these platforms, the collection of users' personal data is inevitable for the completion of any transaction. In many cases, the amount of personal information necessary to get started creates an intrinsic barrier in users' onboarding process, since the growing concern for online privacy might reduce users' willingness to share personal information [4].

In this study, we are interested in understanding the effects of monetary incentives on users' onboarding process at peer-to-peer car sharing platforms, where users have to provide sensitive information such as the registration information of a car and/or a digital copy of a driver's license to engage in any sharing activities.

There has been a long-lasting debate regarding the role of monetary incentives (financial reward) in motivating the disclosure of users' personal information: some have argued that monetary incentives have positive effects on encouraging users to share more personal data with the merchandise [5], although the effect size varies depending on the amount of the monetary incentive [6]; others have found that the benefits of price discounts and personalized service are not effective in mitigating consumers' privacy concerns [7]; or can even unexpectedly intensify the concerns of privacy [8].

In light of these conflicting views and findings, we conduct a randomized field experiment at a large peerto-peer car sharing platform by exogenously varying the amount of the incentive received by new users at the platform. Note that in the context of a peer-to-peer car sharing platform, new users experience at least two stages in their onboarding process: registration on the 
service and booking a car. Here, we seek to understand the effects of monetary incentives in both stages.

Our empirical analysis demonstrates that monetary incentives work no better than non-monetary incentives, such as email reminders, in motivating users to disclose personal information and engage in transaction on the platform. Such finding contributes to the growing literature on the role of monetary incentives on user behavior on digital platforms, and shed new light on the design and implementation of the various incentive schemes.

The rest of this paper is organized as follows. The next section reviews the related literature, which is followed by a brief introduction of the focal digital platform and the experimental design. Section 4 presents the empirical analyses and results. Section 5 discusses our main findings and their implications, and outlines the directions for future work.

\section{Related Literature}

\subsection{Monetary incentives and privacy concerns}

According to the Oxford standard dictionary, an incentive is "a thing that motivates or encourages someone to do something". Incentives can be monetary rewards (e.g., cash bonus or discount for desired behaviors) or non-monetary rewards (e.g., tangible recognition among peers, personalized services). Prior research across different domains has documented the effects of monetary incentives on modifying behavior [9]. For example, prepaid monetary incentives have shown to have a strong positive impact on the response rate in mail surveys [10], and store credits or coupons towards the next purchase are proven to be an effective sales promotion device $[11,12]$.

Drawing upon theories of self-disclosure, prior research in the context of e-commerce demonstrates that monetary incentives can be used as an effective means to induce consumers to release more personal information [13]. For instance, using a controlled lab experiment where subjects were exposed to different levels of monetary incentives ( $\$ 0, \$ 10$, and \$20), Hann et al. [6] found that individuals' decisions regarding information disclosure depend on whether the monetary reward exceeds a certain threshold level. In another notable example, Beresford et al. [14] demonstrated through a field experiment that the vast majority of users did not hesitate in providing personal information, such as monthly incomes, in return of a price discount of $€ 1$ in their DVD purchases, suggesting that monetary incentives can effectively mitigate consumers' privacy concerns.
It is worth noting that in many cases, the motivation for introducing a monetary incentive may be different from how a user perceives it, and it is important to align users' perception with the purpose of the incentive scheme. For instance, in a controlled lab experiment with student subjects, Andrade et al. [8] found that, contrary to the conventional view, monetary reward intensifies privacy concerns. Further investigation suggests that a significant proportion of the subjects considered the monetary reward "as decoys, which companies employ to inveigle individuals to reveal sensitive personal information" [8]. Such finding underscores the necessity to account for the confounding factors within the empirical context when examining the role of monetary incentives in shaping users' information disclosure behavior. In the case of Andrade et al. [8], it is likely that the promised $\$ 10$ check is perceived differently than coupons or price discounts (i.e., the latter clearly conveys the promotion nature).

With this in mind, we adopt an experimental approach to explore the role of monetary incentives on users' information disclosure behavior in the online environment. Unlike prior studies, which are largely focused on the role of coupons or price discounts in the traditional retailing sector, we have chosen to examine the effects of monetary incentives on user onboarding in a novel digital sharing platform.

\subsection{Digital Sharing Platforms}

While sharing of goods and services dates back to the beginning of the human history, the key enabler to those platforms operating at large-scale has been the continuous development of information and communication technologies [15].

The sharing economy is structured around the disintermediation of conventional channels of commerce in the exchange of products and services [16]. As such, there are two guiding principles that need to be taken into account when defining a sharing economy company. First, its business model is based on access, not on ownership. Second, it is grounded on crowd-based networks [17].

More specifically, platforms in the sharing economy:

- Connects people that would not otherwise do business together.

- Create economies of scale to bring together supply and demand.

- Mediate in the relationship between supply and demand, and create a trustworthy environment. 
The emerging sharing platforms have disrupted many industries, which in turn led to significant changes in many aspects of our life. Take the carsharing platforms for example. Recent studies [18] have found that peer-to-peer sharing of cars enabled by such platforms is associated with significant reduction in miles travelled, gas consumption, and car ownership.

Given the socioeconomic significance, it is important to understand how different incentive schemes affect users' onboarding process, which is crucial to the viability and success of sharing platforms. Here, we seek to gain a comprehensive understanding of users' behavior during the initial onboarding process under different incentive schemes, and offer useful implications for the practical design of sharing platforms.

\section{The Field Experiment}

\subsection{CarShare}

CarShare (a pseudonym), the company we have partnered with in this study, is one of the oldest online peer-to-peer car-sharing platforms in Europe. Originating in the early 2000 's, when five neighbors decided to share a car, the company has now grown into a major player in the car-sharing business.

At CarShare, a booking is accepted immediately when the requested car is available and the necessary insurance is arranged. This removes the inherent barrier for renting a car between peers, and thereby makes it convenient for users to share their cars. The ultimate goal of CarShare is to reduce the need for additional cars (and thereby contribute to the energy and environmental targets) by facilitating users to share their cars, optimizing the use of cars.

\subsection{Experimental Design}

To understand the impact of incentives in the sharing economy, we conducted a randomized field experiment on new users of CarShare from April to July in 2016. The experiment was seamlessly integrated in the user onboarding processes, focusing on the communication of incentives via email reminders sent to the new users on the platform.

Users who would like to rent a car on the platform, would need to provide personal details such as name, gender, age, address and telephone number, along with a digital copy of their driver's license. In other words, the requirement of personal data is the main hurdle to user onboarding.
To motivate users to disclose their personal information, and make their first bookings on the platform, we incorporate both monetary as well as non-monetary incentives.

The monetary incentives include discount coupons of different values, which can be redeemed upon firsttime booking. The non-monetary incentive is in the form of a simple reminder (see Appendix for more details). The subjects in this experiment were new users who had already signed up, but not completed their profile (name, gender, address) after 24 hours since registration. They were randomly assigned to the following groups:

- Control: Subjects within this group did not receive any treatment and is solely used for control.

- Group 1: Subjects within this group received an email with a reminder to complete their profiles.

- Group 2: Subjects within this group received an email with a reminder to complete their profiles, and a discount coupon of $€ 5$ for their first trips.

- Group 3: Subjects within this group received an email with a reminder to complete their profiles, and a discount coupon of $€ 10$ for their first trips.

The emails for both Group 2 and 3 contained a discount coupon code that users could redeem immediately upon completing their profiles. Since booking a car is only possible with a completed profile, the introduction of coupon codes would not affect the user on-boarding process, i.e., it is not possible for any user to bypass the self-disclosure stage and redeem the coupon in the booking stage.

The experiment ran over the course of 10 weeks. On each day, the system performed an automatic check on the new sign-ups to validate whether they had already completed their profile within 24 hours, and if not, these users would be randomly assigned to one of the four treatment groups. This process was automated using heuristics that ran every morning on a relational database management system.

To measure the response of users towards the email, both the click-through-rate as well as the openrate were tracked. The email contained a tracking pixel, which triggered a counter when the email was opened. Click-through was tracked on the only button in the email, guiding users to complete their profile.

We first conducted a trial experiment to see if there were errors in the emails, targeting or elsewhere. This was important, since everything that runs live on a company environment is prone to errors. No errors were identified and the data from the trial experiment were included in the final data-set of our study.

We ensured that all the users within their treatment groups received the email reminders. When an email 
was sent successfully, this was logged and could be used to get matched against the user ID, and exclude any user who did not receive the full treatment.

Concurrently, we also identified 35 instances where the account was not created by a human, which were classified as spam, and were removed from the dataset.

\section{Data and Empirical Analysis}

Over the 10-week experiment period, we observed a total of 2315 sign-ups1, where 1360 users either immediately provided the required information during the initial registration process or did so within 24 hours. The remaining 955 users did not provide the required personal information after the first 24 hours upon registration and, thereby, were randomly assigned to the four treatment groups described above.

To obtain an initial understanding of the population in the dataset, we first conducted some descriptive analysis. Tables 1 and 2 summarize the demographic information of the 955 subjects.

As can be seen from Table 1, among the users who disclosed their personal information, most (48.8\%) are between the ages 18-55, roughly equally distributed across age-groups, while only $6.8 \%$ of the population of our study is above 55 years old.

Table 1. Age Distribution

\begin{tabular}{|c|c|c|}
\hline Age & Frequency & Percentage \\
\hline $\mathbf{1 8}-\mathbf{2 5}$ & 122 & 12.7 \\
\hline $\mathbf{2 6}-\mathbf{3 5}$ & 139 & 14.6 \\
\hline $\mathbf{3 6}-\mathbf{4 5}$ & 97 & 10.2 \\
\hline $\mathbf{4 6 - 5 5}$ & 108 & 11.3 \\
\hline $\mathbf{5 6}-\mathbf{6 5}$ & 47 & 4.9 \\
\hline $\mathbf{6 5}+$ & 18 & 1.9 \\
\hline Total & 531 & 55.6 \\
\hline Missing & 424 & 44.4 \\
\hline Total & 955 & 100.0 \\
\hline
\end{tabular}

Overall, $44.4 \%$ of the subjects in our study (424 users) did not reveal their age information. Further, as shown in Table 2, the gender distribution of our subjects in the study is $18.2 \%$ females and $24.1 \%$ males, while more than half of the subjects in our study (550 users or $57.6 \%$ ) did not provide their gender information. As mentioned above, new users do not need to provide their age, gender or driving license upon sign-up, however, such information is necessary to activate the account at CarShare and rent a car.
Table 2. Gender Distribution

\begin{tabular}{|c|c|c|}
\hline Gender & Frequency & Percent \\
\hline Missing & 550 & 57.6 \\
\hline Female & 175 & 18.3 \\
\hline Male & 230 & 24.1 \\
\hline Total & 955 & 100.0 \\
\hline
\end{tabular}

Table 3 presents an overview of the experiment results with respect to users' self-disclosure. Overall, 239 users were assigned to the control Group, among which 41 provided personal data; 240 users were assigned to Group 1 (simple reminder), among which 51 disclosed personal information; 246 users were assigned to Group 2 (simple reminder $+€ 5$ ), among which 53 disclosed personal information; and 230 users were assigned to Group 3 (simple reminder + $€ 10)$ where 47 of them disclosed personal information. Overall, approximately $80 \%$ of the subjects did not provide their personal data.

Table 3. Users' Self-Disclosure

\begin{tabular}{|c|c|c|c|}
\hline $\begin{array}{c}\text { Treatment } \\
\text { Condition }\end{array}$ & $\begin{array}{c}\text { Details not } \\
\text { provided }\end{array}$ & $\begin{array}{c}\text { Details } \\
\text { provided }\end{array}$ & Total \\
\hline Control & 198 & 41 & 239 \\
\hline Reminder & 189 & 51 & 240 \\
\hline $\begin{array}{c}\text { Reminder } \\
+\boldsymbol{\epsilon 5}\end{array}$ & 191 & 55 & 246 \\
\hline $\begin{array}{c}\text { Reminder } \\
+\boldsymbol{€ 1 0}\end{array}$ & 182 & 48 & 230 \\
\hline Total & 760 & 195 & 955 \\
\hline
\end{tabular}

Since only about $20 \%$ of the subjects in the focal population (i.e., the 955 users who did not provide the required information after the first 24 hours upon registration) provided their personal information such as age and gender, we were not able to run a full randomization check. Nevertheless, a post-experiment check shows that among the users who disclosed themselves under the control and three treatment conditions, the age distribution is quite similar to that of the users who provided these details spontaneously (see results from Table 1 and Table 4).

Table 4. Post-Experiment Randomization Check

\begin{tabular}{|c|c|c|c|c|}
\hline Age & Control & Group 1 & Group 2 & Group 3 \\
\hline $\mathbf{1 8}-\mathbf{2 5}$ & 32 & 26 & 27 & 29 \\
\hline $\mathbf{2 6}-\mathbf{3 5}$ & 37 & 36 & 33 & 27 \\
\hline $\mathbf{3 6}-\mathbf{4 5}$ & 24 & 25 & 26 & 25 \\
\hline $\mathbf{4 6}-\mathbf{5 5}$ & 26 & 26 & 31 & 22 \\
\hline $\mathbf{5 6}-\mathbf{6 5}$ & 10 & 10 & 13 & 12 \\
\hline $\mathbf{6 5}+$ & 5 & 4 & 3 & 2 \\
\hline
\end{tabular}

\footnotetext{
${ }^{1}$ We have filtered out the accounts created by spammers based on
} the email address provided during the sign-up process. 
To test the effects of incentives (reminder email and discount coupons), we performed a series of Chisquare tests to determine whether there was any significant difference between different treatments in terms of information disclosure.

Specifically, we compared the disclosure rate between each of the three treatment groups and the control group: i) no reminder (control) vs. a simple reminder ( $p$-value $=0.25)$, ii) no reminder (control) vs. a reminder plus a $€ 5$ discount ( $\mathrm{p}$-value $=0.15)$, iii) no reminder (control) vs. a reminder plus a $€ 10$ discount (p-value $=0.30$ ), as well the disclosure rate between non-monetary treatment, low-monetary treatment and high-monetary treatment. No significant difference was found in terms of disclosure rate.

Table 5. Comparison of Users' Self-Disclosure Rate with Chi-square Test

\begin{tabular}{|c|c|c|c|}
\hline & Control & Group 1 & Group 2 \\
\hline $\begin{array}{c}\text { Group 1: } \\
\text { Reminder }\end{array}$ & 0.25 & & \\
\hline $\begin{array}{c}\text { Group 2: } \\
\text { Reminder } \\
+\boldsymbol{€ 5}\end{array}$ & 0.15 & 0.77 & \\
\hline $\begin{array}{c}\text { Group 3: } \\
\text { Reminder } \\
+\boldsymbol{€ 1 0}\end{array}$ & 0.30 & 0.92 & 0.69 \\
\hline
\end{tabular}

In addition to users' information disclosure, we are also interested in the impact of different incentives on the conversion rate of actual booking. Table 6 presents an overview of the conversion regarding account activation. 17 users $(7.1 \%)$ from the control group made the booking during the experiment period. Among the three treatment groups, the simple reminder treatment (Group 1) yields the highest conversion rate $(11.7 \%)$.

Table 6. Users' Account Activation

\begin{tabular}{|c|c|c|c|}
\hline $\begin{array}{l}\text { Treatment } \\
\text { Condition } \\
\end{array}$ & $\begin{array}{c}\text { Account not } \\
\text { activated }\end{array}$ & $\begin{array}{c}\text { Account } \\
\text { activated }\end{array}$ & Total \\
\hline Control & 222 & 17 & 239 \\
\hline Reminder & 212 & 28 & 240 \\
\hline $\begin{array}{l}\text { Reminder } \\
+€ 5\end{array}$ & 226 & 20 & 246 \\
\hline $\begin{array}{l}\text { Reminder } \\
+€ 10\end{array}$ & 204 & 26 & 230 \\
\hline Total & 864 & 91 & 955 \\
\hline
\end{tabular}

\footnotetext{
2 When we group the subjects from Group 1 and Group 3 and compare the conversion rate between this merged group with control group, we get a p-value of 0.07 .
}

Following a similar procedure as above, we also test whether the observed differences in conversion rate shown in Table 6 are statistically significant. Table 7 presents the results of the Chi-square tests. Although the differences are not statistically significant at the traditional level of $p \leq 0.05$, when comparing Group 1 (simple reminder) and Group 3 (reminder plus a $€ 10$ discount) with the control group, we do observe marginally significant difference, i.e., users who received a simple reminder or a reminder with a $€ 10$ discount coupon were more likely to make bookings on the platform 2 . On the contrary, subjects in Group 2 (simple reminder plus a $€ 5$ discount) were no different than those in the control group in terms of their actual booking behavior.

Table 7. Comparison of Users' Account Activation with Chi-square Test

\begin{tabular}{|c|c|c|c|}
\hline & Control & Group 1 & Group 2 \\
\hline $\begin{array}{c}\text { Group 1: } \\
\text { Reminder }\end{array}$ & 0.08 & & \\
\hline $\begin{array}{c}\text { Group 2: } \\
\text { Reminder } \\
+\boldsymbol{€ 5}\end{array}$ & 0.67 & 0.19 & \\
\hline $\begin{array}{c}\text { Group 3: } \\
\text { Reminder } \\
+\mathbf{\epsilon 1 0}\end{array}$ & 0.11 & 0.90 & 0.24 \\
\hline
\end{tabular}

Finally, we also checked whether the conversion rate conditional on users' self-disclosure is different across the four groups. Tables 8 and 9 summarize these findings. Specifically, while none of the treatment groups show any significant difference from the control group (conditional conversion rate is $41 \%$ ), a low monetary incentive seems to perform worse than a simple email reminder ( $\mathrm{p}$-value $=0.05$ ) and a high monetary incentive $(\mathrm{p}$-value $=0.07)$.

Table 8. Users' Account Activation Conditional on Self-Disclosure

\begin{tabular}{|c|c|c|c|}
\hline $\begin{array}{c}\text { Treatment } \\
\text { Condition }\end{array}$ & $\begin{array}{c}\text { Account not } \\
\text { activated }\end{array}$ & $\begin{array}{c}\text { Account } \\
\text { activated } \\
(\%)\end{array}$ & Total \\
\hline Control & 24 & $17(41 \%)$ & 41 \\
\hline Reminder & 23 & $28(55 \%)$ & 51 \\
\hline $\begin{array}{c}\text { Reminder } \\
+\boldsymbol{€ 5}\end{array}$ & 35 & $20(36 \%)$ & 55 \\
\hline $\begin{array}{c}\text { Reminder } \\
+\boldsymbol{€ 1 0}\end{array}$ & 22 & $26(54 \%)$ & 48 \\
\hline Total & 104 & $91(47 \%)$ & 195 \\
\hline
\end{tabular}


Table 9. Comparison of Users' Account Activation Conditional on Self-Disclosure with Chi-square Test

\begin{tabular}{|c|c|c|c|}
\hline & Control & Group 1 & Group 2 \\
\hline $\begin{array}{c}\text { Group 1: } \\
\text { Reminder }\end{array}$ & 0.20 & & \\
\hline $\begin{array}{c}\text { Group 2: } \\
\text { Reminder } \\
+\boldsymbol{\epsilon 5}\end{array}$ & 0.61 & 0.05 & \\
\hline $\begin{array}{c}\text { Group 3: } \\
\text { Reminder } \\
+\mathbf{\epsilon 1 0}\end{array}$ & 0.23 & 0.94 & 0.07 \\
\hline
\end{tabular}

At the outset, the results from Table 7 and Table 9 suggest a U-shaped effect of monetary incentives, that is, a low level of monetary incentive may be worse than a non-monetary incentive and a high level of monetary incentive in motivating users to actively engage in the transactions on the platform.

\section{Discussion}

In this paper, we examine the impact of different incentives on user onboarding behavior using a randomized field experiment at a large peer-to-peer sharing platform. We find that contrary to the conventional view, coupons or financial rewards is ineffective in motivating users to disclose their personal information in the initial onboarding stage.

However, as soon as the users move to the next stage in their onboarding journey, a simple reminder email can work as effectively as or even better than monetary incentives in persuading them to convert to active users. To the best of our knowledge, this is the first empirical study that directly compares the effects of different incentives (monetary vs. non-monetary) on users' privacy disclosure and actual behavior with an experimental design seamlessly incorporated in the onboarding process of a real-world company.

Our findings need to be interpreted with caution. Reminder emails have been proven useful in this study for users to activate their account. However, including additional price discount in the reminder emails does not necessarily improve such conversion. This particular finding contradicts the results of the extant literature (e.g., [5] and [14]), and is also distinct from the results reported in [8], where the provision of a reward unexpectedly intensified the privacy concern.

Note that the authors of [8] posited that such negative effect of financial reward might be, from the perspective of subjects (students), due to inadequate compensation for disclosing sensitive information. In a similar vein, the insignificant effect associated with the monetary incentives in our study may be attributed to the low perceived value of the monetary compensation for personal data. Specifically, the discount of $€ 5$ or $€ 10$ may only constitute a very small portion of the total rent of a car for a certain period (for instance for a week). Therefore, such monetary incentive could not render any stronger effects than a simple reminder email in encouraging users to disclose personal information.

Second, in the context of car sharing, it might be much more sensible for customers to provide their driving license information during booking instead of the registration process; when customers are already determined to initiate a car booking, a small discount, when compared to a reminding email, might be only considered as icing on the cake, but not the sufficient incentive to induce different behavior.

\subsection{Implications}

From the theoretical perspective, while our study cannot reconcile the inconsistent findings of the extant literature on monetary incentive and privacy disclosure, it provides strong evidence for users' unwillingness to trade off personal identifiable information with a low purchase discount. Such finding suggests the indirect psychological effect associated with monetary incentives may work in the opposite direction to the direct price effect and thereby crowd out the desired behavior (i.e., disclosure of personal information) at the sharing platform. In this regard, the findings from the current study bear some similarity with the results from [19] where the authors demonstrate that while effective in inducing larger volumes of reviews, monetary incentives are less effective than social norms (non-monetary incentives) in generating long reviews. In light of such findings, a promising way to improve the conversion rate during the on-boarding process could be a combination of monetary incentive and social norm. A full treatment of this topic is beyond the scope of our current paper and we will leave it for future work. Our findings also provide implication for practice, as we provide managers with novel insights for better understanding user behavior in the sharing economy, and into the mechanisms that contribute to the onboarding of new users. Further, we demonstrate that users' sensitivity to incentives differs across different stages in the onboarding process. In particular, when they have established the initial relationship (e.g., disclose their personal information) with the platform, a simple email reminder (non-monetary incentive) can work as effectively as monetary incentives in motivating the users to further engage in transactions at the platform. This is good news for many start-ups in the sharing economy, as user-onboarding is vital to the survival 
and success of those companies but they typically face tight budget constraints.

\subsection{Limitations}

The current study bears several limitations. To start with, the measurement of email opening is not perfect, although it has been widely adopted in the majority of randomized field experiments that leverage emails to assign treatments. The reason is that all emails were sent to the registered users before a loyal relationship was established between the platform and those users, some email clients might block the images containing the tracking pixel, and thereby not all opens could be tracked. In other words, only the connection between the treatment and the final action (disclosure of personal information and booking) could be reliably tracked. It is possible that some observed conversion in the treatment groups is purely voluntary (e.g., users who did not open the email but returned to complete their profile after 24 hours of registration) and not driven by the assigned treatments.

Second, the fact that emails were sent at the same time each day could also be a weakness, given that some users received their email later than others compared to the moment of signup.

Finally, while we have a reasonably large sample, since we do not observe all the demographic information from the entire sample of users, it is not possible to discover nuances in users' privacy disclosure. This also limits our ability to interpret the counterintuitive findings from the experimental data.

\subsection{Future Work}

In the current paper, we have primarily focused on the effect of monetary incentives on users' information disclosure and short-term engagement (i.e., first-time booking) at digital car-sharing platforms. Future research can incorporate non-monetary incentives such as social norms [19] to further understand users' sharing behavior. Further, it is worthwhile to dig into the long-term effect of various incentives.

To this point, our current study serves as a useful starting point, and our empirical setup provides rich opportunities to explore interesting phenomena and design problems in the emerging digital sharing platforms.

\subsection{Concluding Remarks}

In this paper, we examine the impact of monetary incentives on user onboarding at digital sharing platforms. We demonstrate that monetary incentives work no better than non-monetary incentives (e.g., a simple email reminder) in encouraging users to disclose personal information and to actively participate in the transactions of peer-to-peer sharing platforms.

\section{References}

[1] Y. Bakos, "The emerging role of electronic marketplaces on the Internet", Communications of the ACM, 41(8), 1998, pp. 35-42.

[2] T.D. Chen, and K.M. Kockelman, "Carsharing's lifecycle impacts on energy use and greenhouse gas emissions," Transportation Research Part D: Transport and Environment, 47, 2016, pp. 276-284.

[3] E.W. Martin, and S.A. Shaheen, "Greenhouse gas emission impacts of carsharing in North America," IEEE Transactions on Intelligent Transportation Systems, 12(4), 2011, pp. 1074-1086.

[4] A. Acquisti, L. Brandimarte, and G. Loewenstein. "Privacy and Human Behavior in the Age of Information," Science, 347(6221), 2015, pp. 509-514.

[5] H. Zhu, C. X. Ou, W.J.A.M van den Heuvel, and H. Liu, "Privacy calculus and its utility for personalization services in e-commerce: An analysis of consumer decisionmaking", Information \& Management, 54(4), 2017, pp. 427-437.

[6] I.H. Hann, K.L. Hui, T. Lee, and I. Png, “Online information privacy: Measuring the cost-benefit trade-off", in ICIS 2002 proceedings, available online at: http://aisel.aisnet.org/icis2002/1.

[7] S. Ward, K. Bridges, and B. Chitty, "Do Incentives Matter? An Examination of On-line Privacy Concerns and Willingness to Provide Personal and Financial Information", Journal of Marketing Communications, 11(1), 2005, pp. 21-40.

[8] E.B. Andrade, V. Kaltcheva, and B. Weitz, "Selfdisclosure on the web: The impact of privacy policy, reward, and company reputation", Advances in Consumer Research, 29, 2002, pp. 350-353.

[9] U. Gneezy, S. Meier, and P. Rey-Biel, "When and why incentives (don't) work to modify behavior," The Journal of Economic Perspectives, 25(4), 2011, pp. 191-209.

[10] J. David, J. Saunders, and V. Mitchell, "Prepaid monetary incentive effects on mail survey response," Journal of Business Research, 57(1), 2004, pp. 21-25.

[11] S.A. Neslin, C. Henderson, and J. Quelch, "Consumer promotions and the acceleration of product purchases", Marketing Science, 4(2), 1985, pp. 147-165. 
[12] D.R. Lichtenstein, R.G. Netemeyer, and S. Burton, "Distinguishing coupon proneness from value consciousness: An acquisition-transaction utility theory perspective", The Journal of Marketing, 54(3), 1990, pp. 54-67.

[13] K.L. Hui, H.H. Teo, and S.Y.T. Lee, "The value of privacy assurance: an exploratory field experiment”, MIS Quarterly, 31(1), 2007, pp. 19-33.

[14] A.R. Beresford, D. Kübler, and S. Preibusch, "Unwillingness to pay for privacy: A field experiment", Economics Letters, 117(1), 2012, pp. 25-27.

[15] G. Zervas, D. Proserpio, and J. W. Byers, "The rise of the sharing economy: Estimating the impact of Airbnb on the hotel industry," Journal of Marketing Research, forthcoming.

[16] F. Bardhi, and G.M. Eckhardt, "Access-based consumption: The case of car sharing", Journal of Consumer Research, 39(4), 2012, pp. 881-898.

[17] A. Sundararajan, The sharing economy: The end of employment and the rise of crowd-based capitalism, MIT Press, 2016.

[18] R. Cervero, A. Golub, and B. Nee, "City CarShare: Longer-Term Travel Demand and Car Ownership Impacts," Transportation Research Record: Journal of the Transportation Research Board, 1992(1), 2007, pp. 70-80.

[19] G. Burtch, Y. Hong, R. Bapna, and V. Griskevicius, "Stimulating online reviews by combining financial incentives and social norms," Management Science, 2017, Forthcoming.

\section{Appendix}

Automatic email sent upon initial registration ${ }^{3}$ :

\section{SUBJECT:}

FIRSTNAME, please complete your profile!

Dear FIRSTNAME,

Great that you signed up at CarShare! Before you can rent, we want to get to know you a little better. Please $\log$ in to your profile page at CarShare and complete the information. You can also upload your license there so we can verify your data.

Do you have any questions? Feel free to reply to this email, we are glad to help.

LINK TO THE LOG-IN PAGE
Email reminder sent after 24 hours of registration:

\section{SUBJECT:}

Reminder: We want to get to know you better!

Dear FIRSTNAME,

Great that you signed up at CarShare. Before you can rent, we want to get to know you a little better. Please $\log$ in to your profile page at CarShare and complete the information. You can also upload your license there so we can verify your data.

Do you have any questions? Feel free to reply to this email, we are glad to help.

\section{LINK TO THE LOG-IN PAGE}

Email reminder with monetary incentive sent after 24 hours of registration:

\section{SUBJECT:}

$€$ DISCOUNT, for a complete profile!

Dear FIRSTNAME,

Great that you signed up at CarShare. Before you can rent, we want to get to know you a little better. Please $\log$ in to your profile page at CarShare and complete the information. You can also upload your license there so we can verify your data.

Once you complete the profile, you can redeem the following voucher (code: XXXXX) of $€$ DISCOUNT for your first booking. Respond quickly and enjoy your discount!

Do you have any questions? Feel free to reply to this email, we are glad to help.

\section{LINK TO THE LOG-IN PAGE}

\footnotetext{
${ }^{3}$ The emails presented here are English translations of the original ones which were written in the local language (non-English).
} 